\title{
PERFIL PSICOLÓGICO-AFECTIVO DE UNA MUESTRA DE NIÑOS DE LA CALLE DE CEUTA
}

\author{
PSYCHOLOGICAL-AFFECTIVE PROFILE OF A SAMPLE \\ OF STREET CHILDREN OF CEUTA
}

\author{
Sergio Cepero Espinosa* y Francisco Herrera Clavero** \\ Universidad de Granada
}

\section{RESUMEN}

Se pretende conocer el perfil afectivo de una muestra de niños de la calle, superando la limitación conceptual reducida al ámbito social.

Se seleccionó una muestra de 53 menores que cumplían alguno de los siguientes requisitos: a) haber vivido en las calles; b) continuar haciéndolo intermitentemente; c) haber abandonado temporalmente del hogar.

El instrumento utilizado ha sido un cuestionario de construcción propia, de 124 ítems.

Los resultados confirman la existencia de dos "perfiles". El primero, se adapta positivamente a los centros de menores y realiza conductas pro-sociales. El segundo, obtiene peores puntuaciones en todos los factores, siendo los más inadaptados.

Palabras clave: Niños de la calle, perfil afectivo, Ciudad Autónoma de Ceuta.

\section{ABSTRACT}

This research seeks to identify the affective profile of a sample of street children, extending their conceptual limitation beyond the social scope.

A sample of 53 children was chosen according to the following criteria: a) to have lived on the street; b) to continue doing so, c) to have runned away from home.

\footnotetext{
* Doctor en Psicopedagogía por la Universidad de Granada, con línea de investigación y publicaciones en el ámbito de los niños de la calle. Becario de Doctorado del Instituto de Estudios Ceutíes. Becario post-doctoral (2006-2008) del M.E.C. en los Estados Unidos (Columbia University, New York) en la investigación: "U.N. Millennium Project: The Millennium Villages Project”. E-mail: filipenses2001@yahoo.es

** Catedrático de E.U. en el Dpto. de Psicología Evolutiva y de la Educación, de la Universidad de Granada. Doctor en Psicología por la Universidad de Granada y Doctor en Filosofía y Ciencias de la Educación por la UNED, con líneas de investigación y numerosas publicaciones en el ámbito del aprendizaje, la adaptación, el autoconcepto, el currículo, la inmigración, la interculturalidad y la convivencia. Miembro de la Junta Rectora del Instituto de Estudios Ceutíes. E-mail: fherrera@ugr.es
} 
The instrument was a questionnaire of own construction, of 124 items. The results show a first profile of children, adapted to the centres of minors and accomplishing pro-social conducts, and a second profile with worst scores in all the studied factors, being the most vulnerable and in need of for intervention.

Key words: Street children, psychological-affective profile, Autonomous City of Ceuta.

\section{Introducción}

A principios del siglo XX aparecen los primeros estudios históricos, literarios, sociológicos y antropológicos sobre la vida en las calles. La población sin hogar de aquel entonces estaba formada mayormente por vagabundos y trabajadores inmigrantes. Durante los años 50 y 60 , se examinan exclusivamente los factores individuales de las personas sin hogar (características físicas y psicológicas); quienes reciben la etiqueta psiquiátrica de "sujetos psicológicamente desviados" (Van Der Ploeg y Scholte, 1997).

Durante las décadas de los 70 y 80, los factores socioeconómicos (pobreza, recortes en los programas de bienestar, escasez de viviendas asequibles etc.) llenan las páginas de revistas médicas y sociológicas especializadas (Van Der Ploeg y Scholte, 1997).

Sin embargo, con el paso de los años la población de "los sin hogar" se ha convertido en una red mucho más heterogénea en la que tienen cabida familias enteras, jóvenes y adolescentes de ambos sexos, e incluso niños que proceden de familias multi-problemáticas. De ahí, que, actualmente, se crea que para estudiar con éxito este grupo poblacional, deben identificarse los factores de riesgo que predisponen a niños y jóvenes a acudir a las calles a múltiples niveles: individual, grupal y social o comunitario (Shinn y Weitzman, 1990 citados en Van Der Ploeg y Scholte, 1997).

Desafortunadamente, durante todos estos años los esfuerzos de investigación no han guiado a la construcción de un cuerpo sistematizado de conocimientos sobre las personas sin hogar y todavía no existe consenso entre los científicos sociales sobre las aproximaciones conceptuales y metodológicas más efectivas a utilizar.

Por otro lado, la investigación sobre los jóvenes sin hogar ha revelado una carencia de habilidades cognitivas-emocionales; lo cual, aumenta el riesgo de que estos jóvenes desarrollen modelos de comportamiento social desajustados. Algunas características psicológicas relacionadas con los rasgos de personalidad de los jóvenes sin hogar son: baja autoestima, resolución inadecuada de los problemas y atribución causal externa.

Cientos de investigaciones en la literatura internacional se limitan actualmente a estudiar sólo los aspectos sociológicos derivados de la vida en las calles de estos niños y adolescentes. Son todavía muy pocos los estudios que, teniendo en cuenta los datos sociológicos de una muestra dada, amplíen su marco de trabajo conceptual incluyendo factores psicológicos-afectivos tan importantes en el comportamiento de estos niños como: personalidad (autoconcepto, autoestima, autoeficacia), estado de ansiedad y adaptación social; lo cual, es precisamente el objetivo de la presente investigación. 


\section{Método}

\section{Muestra}

La muestra ha sido extraída de cuarenta y tres niños procedentes de dos Centros de $\mathrm{Me}$ nores y de un Piso Tutelado de Menores, todos ellos de Ceuta. Junto a ellos, se incluyen algunos niños que aunque vivían regularmente en la calle, en ese momento, se encontraban en algunos de los centros citados.

Las edades de los menores oscilaron entre los 9 y los 17 años, siendo la media de edad de la muestra de 14,8 años. Sólo cinco niñas participaron en el estudio (9,4\%), por lo que no es posible extrapolar ningún resultado referido a las diferencias de género; no obstante para confirmarlo, se aplicó el análisis binomial para pruebas no paramétricas (SPSS. 12.0 para Windows).

\section{Variables}

Factor I (INADAPTADO / VIOLENTO / DESESPERANZADO): Percepción dolorosa de la vida por parte del niño; por lo que realiza conductas disruptivas y se niega a aceptar las reglas de control social. Su inseguridad le lleva a dañar al adversario en vez de tratar de resolver el problema. Relaciones de poder y de abuso.

Factor II (NEUROIDE): El niño ve a los demás mejores que él mismo, sintiéndose incapaz de ayudarles o hacer cosas positivas en la vida. Esto le genera malestar físico, ansiedad e irritabilidad cuando no se le da la razón. Carece de estrategias mediadoras para resolver pacíficamente los problemas, pero no suele llegar a la violencia.

Factor III (INMADURO): Deseo del menor de que los demás le tengan miedo, pues se ve en desventaja (sobre todo física) ante ellos. No confía en la amistad, mostrando cambios de humor, pesimismo y rebeldía a cualquier tipo de reglas.

Factor IV (IRASCIBLE): Sentimientos de desconfianza hacia los demás, temor a que otros quieran vengarse de él y disfrute del mal ajeno. Impaciente y se obsesiona fácilmente con ideas de las que no puede librarse.

Factor V (INSEGURO): Intenta hacer siempre lo correcto aunque no se sienta reforzado o satisfecho plenamente por ello. Suele resolver los problemas sin necesidad de que aparezcan episodios agresivos o violentos. Si le dieran más oportunidades para demostrar sus cualidades se sentiría mejor; pero nunca se atrevería a ser el líder del grupo o a abrirse totalmente ante los demás, porque en el fondo desconfía de ellos.

Factor VI (ASOCIAL): Niño que no se ha integrado socialmente y que es pesimista respecto a su futuro. Se muestra amable con los demás y es incapaz de contradecirles o enfadarse con ellos, pero en realidad le desagrada la forma en que se comportan.

Factor VII (PESIMISTA/VIOLENTO): Referido al niño que siente que los demás lo han tratado injustamente durante toda su vida. Está demasiado acostumbrado a discutir y pelearse con todo y todos; por lo que la vida apenas le presenta aliciente.

Factor VIII (VALIENTE/TRABAJADOR/INSEGURO): Se refiere al menor que se arrepiente de muchas de las cosas que hizo en su pasado, preocupándole lo que puede ocu- 
rrirle en su futuro. Encuentra refugio y satisfacción en el trabajo bien hecho y en una conducta adecuada; aunque, a veces, deba vencer su miedo e inseguridad.

Factor IX (HIPOCONDRÍACO): Ansiedad obsesiva por todo, se siente cansado y/o enfermo para realizar cualquier actividad y se ve inferior respecto a los demás.

Factor X (COMPAÑERO): Referido al niño que tiene muchos amigos y constantemente está gastando bromas. Mantiene la calma y serenidad en momentos de apuro y no es fácil hacerle cambiar de opinión.

\section{Instrumento}

Al no existir ningún cuestionario estandarizado para nuestro propósito, se construyó uno propio de carácter valorativo. Los participantes debían puntuar en una escala Likert de 1 a 5 , su valoración respecto al enunciado planteado. Para su elaboración, se partió de una serie de tests realizados por autores que han estudiado factores afectivos, conativos o sociales y de personalidad. De sus propuestas de intervención se confeccionaron ciento veinticuatro ítems. Los principales tests consultados han sido los siguientes:

\section{Nivel afectivo}

- Test de Autoconcepto Forma A (AFA): Musitu, G. y Gutiérrez M.

- Cuestionario de Autocontrol Infantil y Adolescente (CAS): Capafóns Bonet, A. y Silva Moreno, F.

- Escala de Ansiedad Manifiesta en Niños Revisada (CMAS-R): Cecil B. R. y Bert O. R.

- Cuestionario de Ansiedad Estado/Rasgo en Niños (STAIC): Spielberger, C. D.

\section{Nivel conativo}

- Inventario de Adaptación de Conducta (IAC): De la Cruz, M. V. y Cordero, A.

\section{Personalidad}

- Cuestionario de Personalidad Eysenck (EPQ): Eysenck, H. J. y Eysenck S. B. G.

- Cuestionario de Análisis Clínico, Adolescentes y Adultos (CAQ): Drug S. E.

- Cuestionario Factorial de Personalidad, Forma C (16PF): Cattell, R. B.

\section{Procedimiento}

Únicamente ganándose la confianza de los niños y jóvenes de la calle se puede tener "garantías" de que hablen de sus vidas de una forma más o menos sincera y participativa. A través de la Asociación Elín (única ONG que asiste a los niños de la calle de Ceuta) el autor pudo contactar diariamente durante nueve meses con los menores de la calle de la Ciudad Autónoma de Ceuta.

Al principio la desconfianza y el recelo eran frecuentes, pero con el tiempo, se tuvo acceso (tras la invitación de los propios menores) a los escondites en los que se refugian, lejos del alcance de los extraños y, sobre todo, de la policía. Del mismo modo, no sólo se hablaba con los niños para establecer relaciones de confianza, sino que se comía (a veces del 
mismo plato y del mismo vaso) y convivía con ellos, y se les visitaba cuando se ponían enfermos o resultaban heridos, cosa bastante frecuente.

Una vez cumplido este tiempo, la firma de otro convenio de colaboración, esta vez con la Consejería de Bienestar Social de la Ciudad Autónoma de Ceuta, permitió entrevistar formalmente a estos menores.

En todos los casos las instrucciones fueron las mismas para todos los participantes, motivándoles para que sus respuestas fuesen sinceras. Se tuvieron también en cuenta, las condiciones éticas y deontológicas requeridas en la investigación humana, dando los participantes su consentimiento, consciente y libremente. La información obtenida será utilizada según los fines previstos y conocidos por las personas investigadas. En todo momento, se les garantizó la protección ante riesgos físicos y mentales, etc.

\section{Análisis estadístico}

El procesamiento estadístico utilizado ha sido en primer lugar, la consecución de la fiabilidad y de la validez factorial del cuestionario valorativo. Del análisis factorial, se han conseguido los diez factores citados anteriormente. Tras estos análisis se ha realizado un análisis de conglomerados $K$ medias, del que se han obtenido dos perfiles bien diferenciados sobre los niños de la calle que componen nuestra muestra.

\section{Resultados}

En las Tablas 1, 2 y 3 aparecen los resultados referidos a la fiabilidad y validez del instrumento, así como el peso de cada conglomerado.

TABLA 1: Fiabilidad del instrumento y análisis factorial de los datos (factores 1-5).

\begin{tabular}{|c|c|c|c|c|c|c|c|c|c|}
\hline \multicolumn{10}{|c|}{ Fiabilidad del Cuestionario psicológico-afectivo: ¡Dime Cómo eres! (I) } \\
\hline Ítems & Factor 1 & Ítems & Factor 2 & Ítems & Factor 3 & Ítems & Factor 4 & Ítems & Factor 5 \\
\hline P11 & 0,819 & P64 & 0,169 & P90 & 0,777 & $\mathrm{P} 118$ & 0,674 & P104 & 0,782 \\
\hline P37 & 0,654 & P48 & 0,123 & P5 & 0,631 & P45 & 0,639 & P71 & 0,715 \\
\hline P87 & 0,524 & P62 & 0,696 & P47 & 0,544 & P112 & 0,621 & P95 & 0,598 \\
\hline P12 & 0,521 & P44 & 0,687 & P13 & 0,530 & P6 & 0,470 & P91 & 0,490 \\
\hline P35 & 0,513 & P93 & 0,526 & P32 & 0,512 & P23 & 0,366 & & \\
\hline $\mathrm{P} 2$ & 0,502 & P57 & 0,499 & P26 & 0,431 & & & & \\
\hline P27 & 0,501 & P80 & 0,494 & \multirow{2}{*}{\multicolumn{6}{|c|}{$\begin{array}{l}\text { Coeficiente de Fiabilidad: } \\
\text { "Alpha" de Crombach = 0,8396 }\end{array}$}} \\
\hline & & P77 & 0,453 & & & & & & \\
\hline
\end{tabular}


TABLA 2: Fiabilidad del instrumento y análisis factorial de los datos (factores 6-10).

\begin{tabular}{|c|c|c|c|c|c|c|c|c|c|}
\hline \multicolumn{10}{|c|}{ Fiabilidad del Cuestionario psicológico-afectivo: ¡Dime Cómo eres! (II) } \\
\hline Ítems & Factor 6 & Ítems & Factor 7 & Ítems & Factor 8 & Ítems & Factor 9 & Ítems & Factor 10 \\
\hline P89 & 0,811 & P39 & 0,706 & P73 & 0,194 & P46 & 0,642 & P40 & $-0,264$ \\
\hline P18 & 0,547 & P55 & 0,688 & P63 & 0,147 & P49 & 0,558 & P8 & $-0,212$ \\
\hline P9 & 0,535 & & & P66 & 0,813 & P74 & 0,483 & P72 & 0,754 \\
\hline P109 & 0,533 & & & P70 & 0,675 & P51 & 0,467 & P7 & 0,576 \\
\hline P110 & 0,527 & & & P22 & 0,667 & \multirow{2}{*}{\multicolumn{4}{|c|}{$\begin{array}{l}\text { Coeficiente de Fiabilidad: } \\
\text { "Alpha" de Crombach = 0,8396 }\end{array}$}} \\
\hline & & & & P54 & 0,483 & & & & \\
\hline
\end{tabular}

TABLA 3: Puntuaciones y peso muestral de los conglomerados finales.

\begin{tabular}{|l|c|c|}
\hline \multirow{2}{*}{$\begin{array}{c}\text { PUNTUACIONES Y PESO MUESTRAL DE LOS } \\
\text { FACTORES AFECTIVOS }\end{array}$} & \multicolumn{2}{c|}{$\begin{array}{c}\text { CONGLOMERADOS FINALES } \\
\text { PARA LOS DOS PERFILES }\end{array}$} \\
\cline { 2 - 3 } & PERFIL 1 & PERFIL 2 \\
\hline Puntuación Factor1:Inadaptado/Violento/Desesperanzado & 0,39852 & $-0,91966$ \\
\hline Puntuación Factor 2: Neuroide & 0,28893 & $-0,66677$ \\
\hline Puntuación Factor 3: Inmaduro & 0,22544 & $-0,52024$ \\
\hline Puntuación Factor 4: Irascible & 0,25032 & $-0,57766$ \\
\hline Puntuación Factor 5: Inseguro & 0,21649 & $-0,49958$ \\
\hline Puntuación Factor 6: Asocial & 0,29514 & $-0,68110$ \\
\hline Puntuación Factor 7: Pesimista/Violento & 0,17739 & $-0,40937$ \\
\hline Puntuación Factor 8: Valiente/Trabajador/Inseguro & 0,19051 & $-0,43965$ \\
\hline Puntuación Factor 9: Hipocondríaco & 0,38801 & $-0,89542$ \\
\hline Puntuación Factor 10: Compañero & 0,09942 & $-0,22943$ \\
\hline PESO MUESTRAL: & $69,77 \%$ & $30,23 \%$ \\
\hline
\end{tabular}

A través de los datos puestos de relieve por el programa estadístico SPSS, se confirma que efectivamente aparecen dos grupos de niños bien diferenciados (Gráfico 1).

En primer lugar, con un peso muestral del $69,7 \%$ encontramos a los niños que configura el Perfil I, quienes comparten la característica común de adaptarse positivamente a la vida en los centros y progresar hacia la realización de conductas pro-sociales. Por el contrario, 


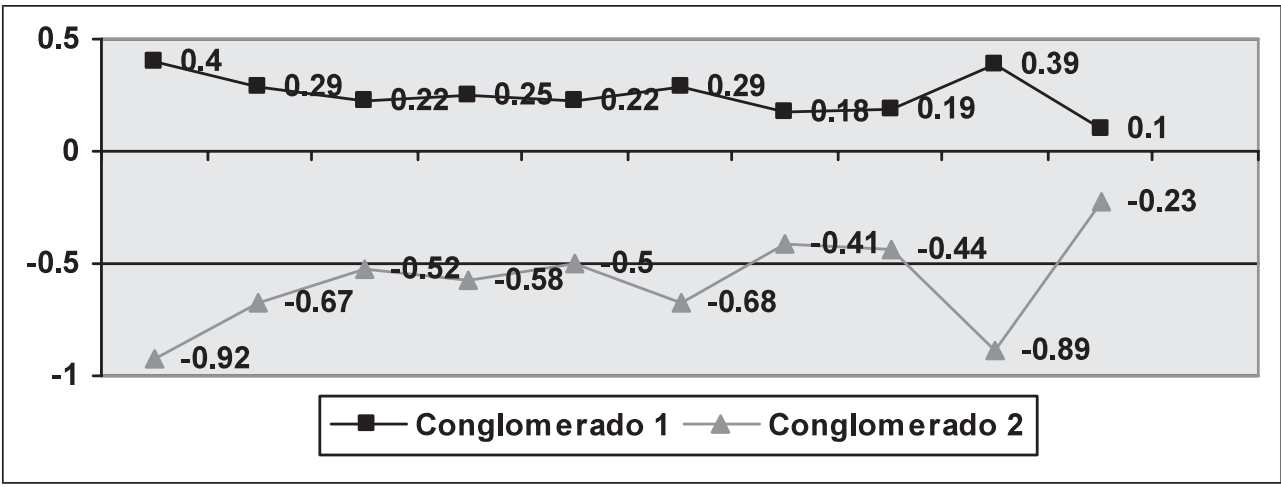

GRÁFICO 1.

Distribución de cada uno de los perfiles de la muestra.

con un peso muestral del 30,2\%, encontramos un segundo grupo de niños (Perfil II), con puntuaciones negativas en todos los factores afectivos, que reclaman un "ritmo" de intervención y de logro de metas diferente respecto al primer perfil.

\section{Conclusiones y discusión}

Resulta cada vez más necesario partir de marcos conceptuales de investigación sólidos, en los que se exploren todas las variables necesarias que permitan ofrecer mejores perspectivas sobre los procesos dinámicos de las personas sin hogar y sobre los factores de riesgo principales que influyen en sus vidas (Van Der Ploeg y Scholte, 1997).

Del mismo modo, es muy poco probable que un único factor (ya sea estructural o individual) sea la causa de la vida en las calles, sino que parece más lógico creer que la vida en las calles obedece a diferentes combinaciones de factores y situaciones, ya que los jóvenes siempre presentan una mezcla conductual. Por ejemplo, Van der Ploeg et al., (1991, citado en Van Der Ploeg y Scholte, 1997), hallaron diferentes combinaciones entre problemas psicológicos [inestable (dependiente, sumiso, enfermizo), retraído (solitario, pesimista, ansioso)] y problemas conductuales (agresión, falta de motivación, búsqueda de sensaciones). En el caso de nuestra investigación sobre los niños de la calle de la Ciudad Autónoma de Ceuta, los diez factores psicológicos estudiados, muestran que no puede intervenirse de la misma forma con los dos perfiles de menores encontrados. Aplicando una única intervención psico-educativa con ellos, siempre quedarán menores a los que no alcancemos y a los que daremos por "imposibles". Ninguna intervención eficaz puede plantearse en términos de no impactar a cada uno de los sujetos a los que va dirigida. Hace falta, sin duda, diseñar programas de intervención "a medida" con cada uno de los dos perfiles de menores. Si nos acercamos a los niños de la calle con instrumentos y actuaciones motivadoras, conocedoras de la realidad que han vivido, aprovechando sus circunstancias vitales, podremos tener éxito en proveerles un futuro digno en el que ellos mismos elijan hasta dónde pueden llegar.

Sólo los programas que se basen en una fuerte revisión y conocimiento sobre el fenómeno de los niños y jóvenes sin hogar, contarán con bases sólidas que aseguren el cambio po- 
sitivo de sus vidas. Así pues, las intervenciones aisladas que no cuenten con el esfuerzo, coordinación y la cooperación del resto de servicios y organizaciones de la comunidad, igualmente producirán resultados insatisfactorios.

Una vez más, la prevención es la clave para erradicar esta tragedia. Además, invertir dinero en prevención permite a las comunidades ahorrar mucho más que en programas de intervención que llegan demasiado tarde para eliminar o reducir los factores de riesgo que empujan a estos niños a una vida de violencia y desajuste social.

Finalmente, la trayectoria evolutiva de riesgo que lleva a los niños y jóvenes a vivir en las calles, puede prevenirse mediante programas de infancia temprana en los que se potencien los factores protectores, al tiempo que se reduzcan los factores de riesgo mucho antes de que se desarrollen conductas problemáticas. Las siguientes orientaciones básicas se presentan a modo de ideas para intervenir en el plano psicológico-afectivo con los niños de nuestra muestra (Herrera Ramírez, 2005):

a) Objetivos básicos:

- Comprender los propios sentimientos y los ajenos.

- Reconocer los sentimientos, clasificarlos, comunicarlos, y asociarlos con comportamientos observables.

- Experimentar sentimientos de confianza y satisfacción por el trabajo realizado.

- Fortalecer el nivel de motivación intrínseca, autoestima, objetividad atribucional, control de la ansiedad y sentimientos de soledad y de suicidio.

b) Metodología:

Aunque pueden usarse muchos programas dentro del área afectiva, destacan aquellos referidos al autoconcepto y desarrollo motivacional (Herrera y Ramírez, 2005).

- Para el desarrollo motivacional las aportaciones más destacadas son:

- Brophy y Kher (1986), referentes a la motivación para aprender.

- Stipek (1988), sobre motivación intrínseca.

- Ames y Ames (1991), en motivación de últimos resultados, tanto por parte del docente como del discente.

- Ames (1992) y Epstein (1989), sobre motivación en metas de logro.

- Montero y Alonso (1992), sobre metas de aprendizaje y metas de logro dentro de los modelos de Covington (1984), Dweck (1986) y Weiner (1986).

- Para el desarrollo del autoconcepto:

Destacar las aportaciones de Coopersmith y Feldman (1980), Machargo (1989) y Alonso (1999), que tienen en cuenta los diferentes aspectos del autoconcepto: emocional, social, personal, físico, familiar y académico.

\section{Referencias bibliográficas}

Alonso, E. (1999). Intervención para la mejora del autoconcepto en alumnos de educación secundaria. Tesis Doctoral. Las Palmas de Gran Canaria: Universidad de Las Palmas de Gran Canaria. Servicio de Publicaciones y Producción Documental. 
Alonso, E. (2001). Intervención para la mejora del autoconcepto en alumnos de educación secundaria. Las Palmas de Gran Canaria: Universidad de Las Palmas de Gran Canaria. Servicio de Publicaciones y Producción Documental.

Asociación Elín. (2001). Proyecto de intervención socio-educativa con niños y jóvenes marroquies en las calles de Ceuta. Manucristo no publicado.

González Leandro, P. y Pelechano, V. (1996). "Programa Integrado de Entrenamiento en Habilidades Interpersonales en el Ciclo Medio". En V. Pelechano (Dir.). Habilidades Interpersonales. Teoría Minima y Programas de Intervención. Vol. II. Valencia: Promolibro.

Herrera, F. y Ramírez, M. I. (2005). "Pluriculturalidad, multiculturalidad e interculturalidad". En D. Castellano, A. Gil y P. Serrano: Mujeres. El análisis (pp. 283-311). Colección año 2468. La mediación social. Universidad Jaime I: Fondo Social Europeo. Proyecto Equal.

Herrera Ramírez, M. I. (2005). ¿Qué ocurre entre el autoconcepto y el rendimiento académico, en un contexto pluricultural? Manuscrito presentado para publicación.

Lalor, K. J. (1999). "Street children: A comparative perspective". Child Abuse and Neglect, 8 (23), 759-770.

Machargo, J. (1989): El profesor y el autoconcepto de sus alumnos. Madrid: Escuela Española.

Mcdonald, P. y Garrow, E. (2000). Reaching children in need: What's being done-what you can do. Eastbourne, Inglaterra: Kingsway Publications.

Monjas, M.I. (1993). Programa de enseñanza de habilidades de interacción social. Para niños y niñas en edad escolar. Valladolid: M.I.M.C.

Monjas, M.I. (1996). Programa de enseñanza de habilidades de interacción social (PEHIS) para niños y niñas en edad escolar. Madrid: Ciencias de la Educación Preescolar y Especial.

Pelechano, V. y González Leandro, P. (1996a). "Programa de entrenamiento en fases de solución de problemas". En V. Pelechano (Dir.). Habilidades Interpersonales. Teoría Mínima y Programas de Intervención. Vol. II. Valencia: Promolibro.

Pelechano, V. y González Leandro, P. (1996b). "Programa de entrenamiento en dimensiones de solución de problemas interpersonales". En V. Pelechano (Dir.). Habilidades Interpersonales. Teoría Minima y Programas de Intervención. Vol. II. Valencia: Promolibro.

Rizzini, I. y Lusk, M. W. (1995). "Children in the streets: Latin America's lost generation”. Children and Youth Services Review, 3 (17), 391-400.

Trianes, M. V. y Muñoz, A. (1994). Programa de Educación Social y Afectiva. Málaga: Puerta Nueva, Delegación de Educación y Cultura.

Van Der Ploeg, J. y Scholte, E. (1997). Homeless youth. Working with children and adolescents series. London: SAGE Publications. 


\section{Cuestionario Afectivo “¿Dime cómo eres!”}

Este cuestionario es anónimo y confidencial, por ello te rogamos que contestes con sinceridad.

Procura responder a todas las frases que se te ofrecen, sin detenerte demasiado en cada una de ellas.

\section{MUCHAS GRACIAS}

Para contestar debes rodear con un círculo la opción que te parezca más portuna, de las cinco posibles que se te ofrecen:
1. Totalmente en desacuerdo
2. Algo en desacuerdo
3. No tengo opinión formada
4. Algo de acuerdo

5. Totalmente de acuerdo

\begin{tabular}{|c|c|c|c|c|c|}
\cline { 2 - 5 } \multicolumn{1}{l|}{} & $\begin{array}{c}\text { Totalmente } \\
\text { en } \\
\text { desacuerdo }\end{array}$ & $\begin{array}{c}\text { Algo en } \\
\text { desacuerdo }\end{array}$ & $\begin{array}{c}\text { No tengo } \\
\text { opinión }\end{array}$ & $\begin{array}{c}\text { Algo de } \\
\text { acuerdo }\end{array}$ & $\begin{array}{c}\text { Totalmente } \\
\text { de acuerdo }\end{array}$ \\
\hline $\begin{array}{l}\text { 1. Me gusta estar en una reunión con mucha } \\
\text { gente }\end{array}$ & 1 & 2 & 3 & 4 & 5 \\
\hline $\begin{array}{c}\text { 2. Cuando tengo mucha sed y es imposible be- } \\
\text { ber en ese momento, sólo pienso en las ga- } \\
\text { nas que tengo de beber }\end{array}$ & 1 & 2 & 3 & 4 & 5 \\
\hline $\begin{array}{l}\text { 3. Soy de las personas a quien los demás sue- } \\
\text { len respetar y pedir consejo }\end{array}$ & 1 & 2 & 3 & 4 & 5 \\
\hline 4. Me "vengo abajo" cuando algo me sale mal & 1 & 2 & 3 & 4 & 5 \\
\hline $\begin{array}{l}\text { 5. Paso de reírme o enfadarme o de estar feliz a } \\
\text { estar triste, sin ninguna razón para ello }\end{array}$ & 1 & 2 & 3 & 4 & 5 \\
\hline $\begin{array}{c}\text { 6. El tiempo que se dedica a planear el futuro, } \\
\text { no sirve para nada }\end{array}$ & 1 & 2 & 3 & 4 & 5 \\
\hline 7. Tengo muchos amigos & 1 & 2 & 3 & 4 & 5 \\
\hline $\begin{array}{l}\text { 8. Soy positivo con la vida y le saco todo el } \\
\text { partido que puedo }\end{array}$ & 1 & 2 & 3 & 4 & 5 \\
\hline 9. Pierdo la paciencia fácilmente & 1 & 2 & 3 & 4 & 5 \\
\hline $\begin{array}{c}\text { 10. Cuando ocurre una emergencia, pienso con } \\
\text { calma y serenidad }\end{array}$ & 1 & 2 & 3 & 4 & 5 \\
\hline 11. Mi vida rebosa alegría y felicidad & 1 & 2 & 3 & 4 & 5 \\
\hline $\begin{array}{c}\text { 12. Esatndo a solas, es como me encuentro a } \\
\text { gusto }\end{array}$ & 1 & 2 & 3 & 4 & 5 \\
\hline $\begin{array}{c}\text { 13. Disfruto realizando trabajos constructivos } \\
\text { que exijan máquinas o herramientas de pre- } \\
\text { cisión }\end{array}$ & 1 & 2 & 3 & 4 & 5 \\
\hline 14. Tengo mala suerte en todo & 1 & 2 & 3 & 4 & 5 \\
\hline
\end{tabular}


Perfil psicológico-afectivo de una muestra de niños...

\begin{tabular}{|c|c|c|c|c|c|}
\hline & $\begin{array}{c}\text { Totalmente } \\
\text { en } \\
\text { desacuerdo }\end{array}$ & \begin{tabular}{c|} 
Algo en \\
desacuerdo
\end{tabular} & $\begin{array}{l}\text { No tengo } \\
\text { opinión }\end{array}$ & $\begin{array}{l}\text { Algo de } \\
\text { acuerdo }\end{array}$ & $\begin{array}{l}\text { Totalmente } \\
\text { de acuerdo }\end{array}$ \\
\hline $\begin{array}{l}\text { 15. Tengo muchas y muy buenas cualidades que } \\
\text { la gente aprecia }\end{array}$ & 1 & 2 & 3 & 4 & 5 \\
\hline $\begin{array}{l}\text { 16. Es divertido ver cómo una pandilla molesta } \\
\text { o mete miedo a un niño pequeño }\end{array}$ & 1 & 2 & 3 & 4 & 5 \\
\hline 17. Tengo pesadillas y me despierto asustado & 1 & 2 & 3 & 4 & 5 \\
\hline $\begin{array}{l}\text { 18. Si algo me da miedo, me aguanto y hago lo } \\
\text { que tengo que hacer }\end{array}$ & 1 & 2 & 3 & 4 & 5 \\
\hline $\begin{array}{l}\text { 19. Cuido mucho las cosas que pertenecen a los } \\
\text { demás }\end{array}$ & 1 & 2 & 3 & 4 & 5 \\
\hline $\begin{array}{l}\text { 20. Una norma segura en esta vida es "no con- } \\
\text { fíes en nadie" }\end{array}$ & 1 & 2 & 3 & 4 & 5 \\
\hline $\begin{array}{l}\text { 21. Puedo aguantar que me peguen o insulten } \\
\text { con tal de hacer algo que creo que debo ha- } \\
\text { cer }\end{array}$ & 1 & 2 & 3 & 4 & 5 \\
\hline 22. Me divierte molestar a los demás & 1 & 2 & 3 & 4 & 5 \\
\hline $\begin{array}{l}\text { 23. Incluso estando acompañado, me siendo solo } \\
\text { y desgraciado }\end{array}$ & 1 & 2 & 3 & 4 & 5 \\
\hline $\begin{array}{l}\text { 24. Cuando me deprimo, tardo bastante en co- } \\
\text { menzar a animarme }\end{array}$ & 1 & 2 & 3 & 4 & 5 \\
\hline 25. No creo estar peor de salud que los demás & 1 & 2 & 3 & 4 & 5 \\
\hline $\begin{array}{l}\text { 26. Me pasan por la mente palabrotas o ideas } \\
\text { sucias y no puedo librarme de ellas }\end{array}$ & 1 & 2 & 3 & 4 & 5 \\
\hline $\begin{array}{l}\text { 27. Cuando visito a alguien o voy a algún sitio } \\
\text { donde hay otros usos y costumbres, me sien- } \\
\text { to perdido }\end{array}$ & 1 & 2 & 3 & 4 & 5 \\
\hline $\begin{array}{l}\text { 28. Si volviera a nacer, haría muchas cosas de } \\
\text { manera diferente }\end{array}$ & 1 & 2 & 3 & 4 & 5 \\
\hline 29. Hago inmediatamente lo que me piden & 1 & 2 & 3 & 4 & 5 \\
\hline 30. Pienso que soy torpe al manejar objetos & 1 & 2 & 3 & 4 & 5 \\
\hline $\begin{array}{l}\text { 31. Tengo poca "voluntad" para cumplir lo que } \\
\text { me propongo }\end{array}$ & 1 & 2 & 3 & 4 & 5 \\
\hline 32. Me gusta hacer rabiar a los animales & 1 & 2 & 3 & 4 & 5 \\
\hline $\begin{array}{l}\text { 33. Me gusta estar con amigos que gastan bro- } \\
\text { mas, aunque éstas molesten a los demás y } \\
\text { puedan ser un poco peligrosas }\end{array}$ & 1 & 2 & 3 & 4 & 5 \\
\hline 34. Me avergüenzo de muchas cosas que hago & 1 & 2 & 3 & 4 & 5 \\
\hline $\begin{array}{l}\text { 35. A pesar de que tengo problemas, como to- } \\
\text { dos, soy una persona feliz y contenta }\end{array}$ & 1 & 2 & 3 & 4 & 5 \\
\hline
\end{tabular}




\begin{tabular}{|c|c|c|c|c|c|}
\hline & $\begin{array}{c}\text { Totalmente } \\
\text { en } \\
\text { desacuerdo }\end{array}$ & $\begin{array}{c}\text { Algo en } \\
\text { desacuerdo }\end{array}$ & $\begin{array}{l}\text { No tengo } \\
\text { opinión }\end{array}$ & $\begin{array}{l}\text { Algo de } \\
\text { acuerdo }\end{array}$ & $\begin{array}{l}\text { Totalmente } \\
\text { de acuerdo }\end{array}$ \\
\hline $\begin{array}{l}\text { 36. Me siendo cansado sin ningún motivo para } \\
\text { ello }\end{array}$ & 1 & 2 & 3 & 4 & 5 \\
\hline $\begin{array}{l}\text { 37. Me vienen ideas a la cabeza que no me dejan } \\
\text { dormir por la noche }\end{array}$ & 1 & 2 & 3 & 4 & 5 \\
\hline $\begin{array}{l}\text { 38. Si he robado algo y me dicen; “¿Por qué lo } \\
\text { has hecho?", no sé qué decir }\end{array}$ & 1 & 2 & 3 & 4 & 5 \\
\hline $\begin{array}{l}\text { 39. La mayoría de las personas son amables y } \\
\text { quieren ayudarme }\end{array}$ & 1 & 2 & 3 & 4 & 5 \\
\hline $\begin{array}{l}\text { 40. Cuando los amigos me gastan una broma, no } \\
\text { me molesta y termino riéndome juntamente } \\
\text { con ellos }\end{array}$ & 1 & 2 & 3 & 4 & 5 \\
\hline 41. La vida es muy triste y dolorosa & 1 & 2 & 3 & 4 & 5 \\
\hline 42. Me gustan las peleas y las riñas & 1 & 2 & 3 & 4 & 5 \\
\hline $\begin{array}{l}\text { 43. Tengo confianza en mí para dar la cara y re- } \\
\text { solver la mayoría de los problemas con los } \\
\text { que me encuentro }\end{array}$ & 1 & 2 & 3 & 4 & 5 \\
\hline $\begin{array}{l}\text { 44. Cuando los demás me dicen que debo por- } \\
\text { tarme bien, les pregunto: “¿Qué quiere decir } \\
\text { eso?" }\end{array}$ & 1 & 2 & 3 & 4 & 5 \\
\hline $\begin{array}{l}\text { 45. Ya estoy harto de todo; la vida no merece la } \\
\text { pena vivirla }\end{array}$ & 1 & 2 & 3 & 4 & 5 \\
\hline $\begin{array}{l}\text { 46. Permanezco en silencio cada vez que una } \\
\text { persona mayor está hablando }\end{array}$ & 1 & 2 & 3 & 4 & 5 \\
\hline 47. Me preocupa que algo malo me pase & 1 & 2 & 3 & 4 & 5 \\
\hline $\begin{array}{l}\text { 48. Los demás piensan de mí que soy muy ale- } \\
\text { gre y animoso }\end{array}$ & 1 & 2 & 3 & 4 & 5 \\
\hline $\begin{array}{l}\text { 49. Cuando tengo problemas prefiero guardár- } \\
\text { melos para mí, antes que contárselos a mis } \\
\text { amigos }\end{array}$ & 1 & 2 & 3 & 4 & 5 \\
\hline $\begin{array}{l}\text { 50. La gente pone reglas para fastidiarme y de- } \\
\text { jarme sin libertad }\end{array}$ & 1 & 2 & 3 & 4 & 5 \\
\hline $\begin{array}{l}\text { 51. Cuando algo me da mucho miedo, no puedo } \\
\text { imaginarme otras cosas que me distraigan }\end{array}$ & 1 & 2 & 3 & 4 & 5 \\
\hline $\begin{array}{l}\text { 52. Hay días en que mi cabeza no trabaja con } \\
\text { tanta claridad }\end{array}$ & 1 & 2 & 3 & 4 & 5 \\
\hline 53. Evito hacer las cosas que no debo & 1 & 2 & 3 & 4 & 5 \\
\hline 54. Siento que me falta el aire & 1 & 2 & 3 & 4 & 5 \\
\hline
\end{tabular}


Perfil psicológico-afectivo de una muestra de niños...

\begin{tabular}{|c|c|c|c|c|c|}
\hline & \\
\hline & $\begin{array}{c}\text { Totalmente } \\
\text { en } \\
\text { desacuerdo }\end{array}$ & $\begin{array}{c}\text { Algo en } \\
\text { desacuerdo }\end{array}$ & $\begin{array}{l}\text { No tengo } \\
\text { opinión }\end{array}$ & $\begin{array}{l}\text { Algo de } \\
\text { acuerdo }\end{array}$ & $\begin{array}{l}\text { Totalmente } \\
\text { de acuerdo }\end{array}$ \\
\hline $\begin{array}{l}\text { 55. Algunas personas creen que les he hecho } \\
\text { una faena y quieren vengarse de mí }\end{array}$ & 1 & 2 & 3 & 4 & 5 \\
\hline $\begin{array}{l}\text { 56. Me cuesta creer que hacer siempre lo que } \\
\text { me apatece pueda perjudicarme }\end{array}$ & 1 & 2 & 3 & 4 & 5 \\
\hline $\begin{array}{l}\text { 57. Siento mucha pena al ver un animal cogido } \\
\text { en un cepo o trampa }\end{array}$ & 1 & 2 & 3 & 4 & 5 \\
\hline $\begin{array}{l}\text { 58. Aunque los demás piensen mal de mí, no me } \\
\text { importa, yo voy a lo mío }\end{array}$ & 1 & 2 & 3 & 4 & 5 \\
\hline $\begin{array}{l}\text { 59. Me gustaría hacer cosas arriesgadas como } \\
\text { montar en una moto muy rápida }\end{array}$ & 1 & 2 & 3 & 4 & 5 \\
\hline $\begin{array}{l}\text { 60. Necesito estar solo para pensar cómo hacer } \\
\text { algo bien. No ncesito ayuda de nadie }\end{array}$ & 1 & 2 & 3 & 4 & 5 \\
\hline 61. Me disculpo cuando digo o hago algo mal & 1 & 2 & 3 & 4 & 5 \\
\hline $\begin{array}{l}\text { 62. Me enfado y soy violento si los demás no } \\
\text { me obedecen }\end{array}$ & 1 & 2 & 3 & 4 & 5 \\
\hline $\begin{array}{l}\text { 63. Comparado con los demás, tengo menos } \\
\text { fuerza y energía }\end{array}$ & 1 & 2 & 3 & 4 & 5 \\
\hline $\begin{array}{l}\text { 64. Odio la idea de tener que ir a un hospital, si } \\
\text { caigo enfermo }\end{array}$ & 1 & 2 & 3 & 4 & 5 \\
\hline 65. Pienso que nadie me quire realmente & 1 & 2 & 3 & 4 & 5 \\
\hline 66. Me siento tranquilo y seguro de mí mismo & 1 & 2 & 3 & 4 & 5 \\
\hline $\begin{array}{l}\text { 67. Cuando me excito pierdo la cabeza y hago } \\
\text { tonterías }\end{array}$ & 1 & 2 & 3 & 4 & 5 \\
\hline $\begin{array}{l}\text { 68. Me meto en más peleas y discusiones que } \\
\text { los demás }\end{array}$ & 1 & 2 & 3 & 4 & 5 \\
\hline $\begin{array}{l}\text { 69. Puedo engañar a los demás mostrándome } \\
\text { amable con ellos, pero en realidad me des- } \\
\text { agrandan }\end{array}$ & 1 & 2 & 3 & 4 & 5 \\
\hline $\begin{array}{l}\text { 70. Aunque me castiguen o me peguen, es difícil } \\
\text { que así consigan algo de mí }\end{array}$ & 1 & 2 & 3 & 4 & 5 \\
\hline 71. Tengo el vientre molesto o hinchado & 1 & 2 & 3 & 4 & 5 \\
\hline 72. Tengo mucha energía cuando la necesito & 1 & 2 & 3 & 4 & 5 \\
\hline $\begin{array}{l}\text { 73. No puedo eviar pensar en por qué mis ami- } \\
\text { gos se comportan de la manera que lo hacen }\end{array}$ & 1 & 2 & 3 & 4 & 5 \\
\hline $\begin{array}{l}\text { 74. La gente elige sus amistades por los benefi- } \\
\text { cios que le den }\end{array}$ & 1 & 2 & 3 & 4 & 5 \\
\hline 75. Me preocupa lo que la gente piense de mí & 1 & 2 & 3 & 4 & 5 \\
\hline
\end{tabular}




\begin{tabular}{|c|c|c|c|c|c|}
\hline & $\begin{array}{c}\text { Totalmente } \\
\text { en } \\
\text { desacuerdo }\end{array}$ & $\begin{array}{c}\text { Algo en } \\
\text { desacuerdo }\end{array}$ & $\begin{array}{l}\text { No tengo } \\
\text { opinión }\end{array}$ & $\begin{array}{l}\text { Algo de } \\
\text { acuerdo }\end{array}$ & $\begin{array}{l}\text { Totalmente } \\
\text { de acuerdo }\end{array}$ \\
\hline $\begin{array}{l}\text { 76. Prefiero que otros dirijan el grupo en el que } \\
\text { estoy y cargen con todas las preocupaciones }\end{array}$ & 1 & 2 & 3 & 4 & 5 \\
\hline $\begin{array}{l}\text { 77. Sé muy bien las cosas importante que quiero } \\
\text { hacer en esta vida }\end{array}$ & 1 & 2 & 3 & 4 & 5 \\
\hline $\begin{array}{l}\text { 78. Al estar en un lugar de silencio o de reposo, } \\
\text { temo que me entren ganas de gritar }\end{array}$ & 1 & 2 & 3 & 4 & 5 \\
\hline $\begin{array}{l}\text { 79. No me importa compartir mis cosas con los } \\
\text { demás }\end{array}$ & 1 & 2 & 3 & 4 & 5 \\
\hline $\begin{array}{l}\text { 80. Tengo dudas de haberle sido útil a alguien } \\
\text { en esta vida }\end{array}$ & 1 & 2 & 3 & 4 & 5 \\
\hline $\begin{array}{l}\text { 81. Me encuentro a gusto conmigo mismo y con } \\
\text { lo que me rodea }\end{array}$ & 1 & 2 & 3 & 4 & 5 \\
\hline $\begin{array}{l}\text { 82. Compruebo una y otra vez si he hecho co- } \\
\text { rrectamente las cosas }\end{array}$ & 1 & 2 & 3 & 4 & 5 \\
\hline $\begin{array}{l}\text { 83. No me siento tan desdichado como para llo- } \\
\text { gar }\end{array}$ & 1 & 2 & 3 & 4 & 5 \\
\hline 84. Me gusta que la gente suela contar conmigo & 1 & 2 & 3 & 4 & 5 \\
\hline 85. Creo que no valgo para nada & 1 & 2 & 3 & 4 & 5 \\
\hline $\begin{array}{l}\text { 86. Mis verdaderos amigos, pueden contarse con } \\
\text { los dedos de una mano }\end{array}$ & 1 & 2 & 3 & 4 & 5 \\
\hline $\begin{array}{l}\text { 87. Últimamente me importa un comino lo que } \\
\text { pueda pasar }\end{array}$ & 1 & 2 & 3 & 4 & 5 \\
\hline $\begin{array}{l}\text { 88. Soporto el dolor y las incomodidades tanto o } \\
\text { más que los demás }\end{array}$ & 1 & 2 & 3 & 4 & 5 \\
\hline $\begin{array}{l}\text { 89. Me molesta no tener libertad y medios para } \\
\text { vivir de otra forma }\end{array}$ & 1 & 2 & 3 & 4 & 5 \\
\hline $\begin{array}{l}\text { 90. Al oír hablar de una enfermedad empiezo a } \\
\text { pensar que tengo algo parecido }\end{array}$ & 1 & 2 & 3 & 4 & 5 \\
\hline $\begin{array}{l}\text { 91. Me gustaría que los demás chicos me tuvie- } \\
\text { sen miedo }\end{array}$ & 1 & 2 & 3 & 4 & 5 \\
\hline $\begin{array}{l}\text { 92. Al recordar los aciertos y fallos de mi vida } \\
\text { pasada, pienso más en los aciertos }\end{array}$ & 1 & 2 & 3 & 4 & 5 \\
\hline 93. Soy muy hábil con mis manos & 1 & 2 & 3 & 4 & 5 \\
\hline $\begin{array}{l}\text { 94. Personalmente siento que la gente ha sido } \\
\text { bastante injusta conmigo }\end{array}$ & 1 & 2 & 3 & 4 & 5 \\
\hline $\begin{array}{l}\text { 95. Me molesta mucho que los mayores me nie- } \\
\text { gen lo que pido }\end{array}$ & 1 & 2 & 3 & 4 & 5 \\
\hline
\end{tabular}


Perfil psicológico-afectivo de una muestra de niños...

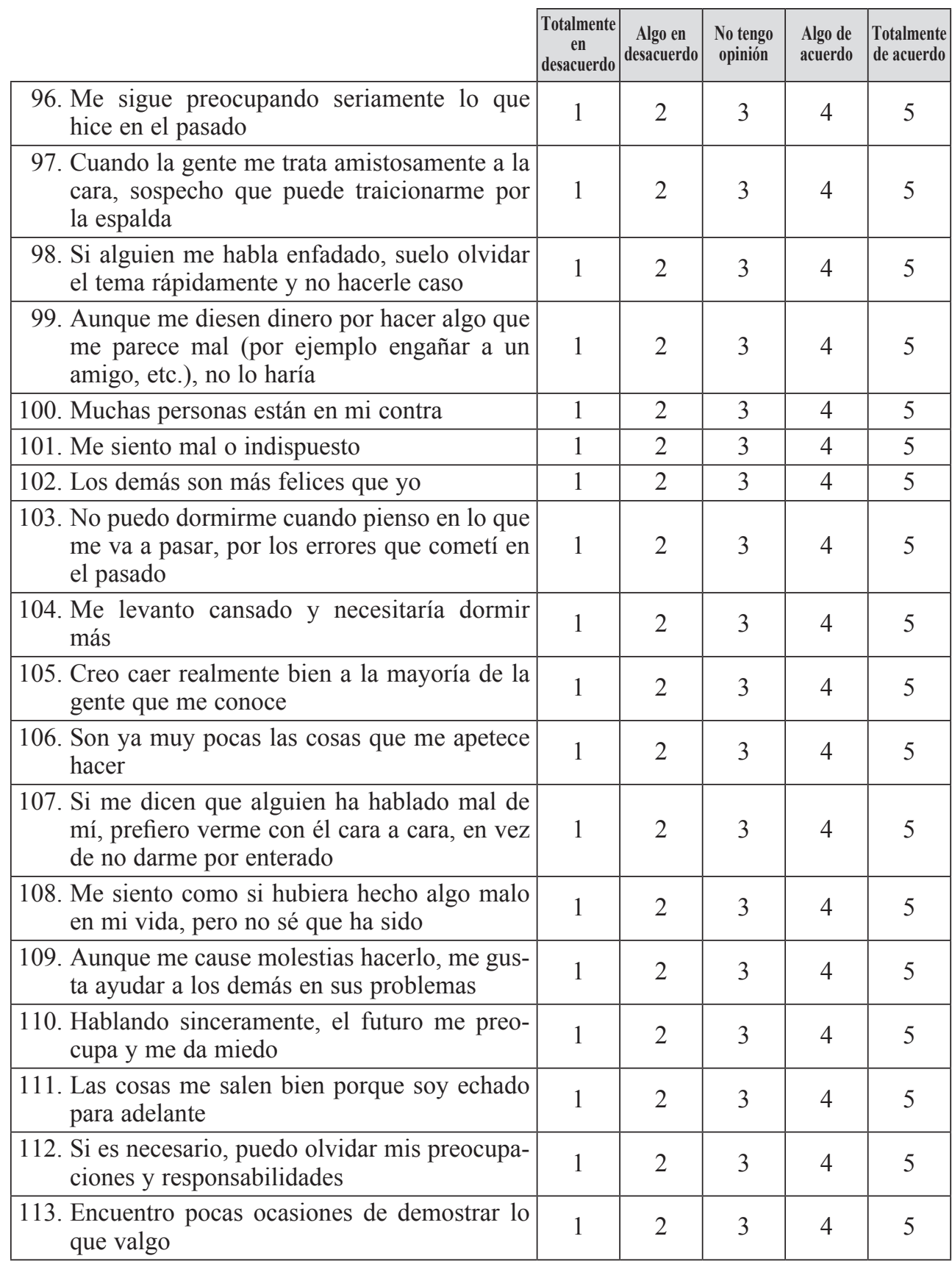


Sergio Cepero Espinosa et al.

\begin{tabular}{|c|c|c|c|c|c|}
\hline & \begin{tabular}{|c|} 
Totalmente \\
en \\
desacuerdo \\
\end{tabular} & $\begin{array}{c}\text { Algo en } \\
\text { desacuerdo }\end{array}$ & $\begin{array}{l}\text { No tengo } \\
\text { opinión }\end{array}$ & $\begin{array}{l}\text { Algo de } \\
\text { acuerdo }\end{array}$ & $\begin{array}{l}\text { Totalmente } \\
\text { de acuerdo }\end{array}$ \\
\hline $\begin{array}{l}\text { 114. Siento que mis nervios están a punto de esta- } \\
\text { llar }\end{array}$ & 1 & 2 & 3 & 4 & 5 \\
\hline 115. Siempre estoy preocupado por algo & 1 & 2 & 3 & 4 & 5 \\
\hline $\begin{array}{l}\text { 116. Todas las personas que conozco me caen } \\
\text { bien }\end{array}$ & 1 & 2 & 3 & 4 & 5 \\
\hline $\begin{array}{l}\text { 117. Mis problemas son tan importantes, que no } \\
\text { puedo olvidarlos ni por un momento }\end{array}$ & 1 & 2 & 3 & 4 & 5 \\
\hline $\begin{array}{l}\text { 118. Si fracaso en algo una vez, es difícil que } \\
\text { vuelva a intentarlo, prefiero darme por ven- } \\
\text { cido }\end{array}$ & 1 & 2 & 3 & 4 & 5 \\
\hline $\begin{array}{l}\text { 119. Cuando hago una tontería, me quedo preocu- } \\
\text { pado mucho tiempo pensando en lo que hice }\end{array}$ & 1 & 2 & 3 & 4 & 5 \\
\hline 120. Me molesta no ser más guapo y atractivo & 1 & 2 & 3 & 4 & 5 \\
\hline $\begin{array}{l}\text { 121. Tengo pocos éxitos en la vida por mi poca } \\
\text { valía }\end{array}$ & 1 & 2 & 3 & 4 & 5 \\
\hline $\begin{array}{l}\text { 122. Me gusta estar rodeado de personas alegres } \\
\text { y entusiastas }\end{array}$ & 1 & 2 & 3 & 4 & 5 \\
\hline $\begin{array}{l}\text { 123. Hago lo que quiero a cada momento del día, } \\
\text { sin obedecer ninguna clase de reglas }\end{array}$ & 1 & 2 & 3 & 4 & 5 \\
\hline
\end{tabular}

